\title{
éditorial
}

\section{La radioactivité naturelle}

\author{
Exposé de J. PRADEL \\ Président de la Société française de radioprotection \\ lors de l'Assemblée générale, Paris, 24 juin 1987
}

La radioactivité naturelle constitue un sujet très vaste qui a été déjà abordé en de nombreuses occasions, notamment lors du Congrès SFRP de Monte-Carlo en $1984[4,16]$. Nous ne devons pas oublier, non plus, la contribution de notre ami Henri FRANÇOIS sous la forme de son livre "La radioactivité, c'est naturel" [6]. C'est pourquoi je me limiterai aujourd'hui à ne traiter que de deux radionucléides primordiaux - uranium 238 et thorium 232 - en laissant de côté le troisième, le potassium 40 . Je ne parlerai pas non plus des rayonnements cosmiques et des radionucléides cosmogéniques associés.

Dans ce domaine ainsi restreint, je me contenterai d'insister sur quatre points essentiels:

1. la terre est une planète dont tous les matériaux présentent une radioactivité naturelle, certes faible, mais aisément mesurable;

2. l'air que nous respirons présente également une radioactivité naturelle très importante;

3. les mineurs d'uranium peuvent être soumis à des expositions très élevées en l'absence de dispositions convenables;

4. on peut essayer d'utiliser, pour fixer les limites en matière de gestion de déchets, les repères que nous fournit la radioactivité naturelle.

\section{RADIOACTIVITÉ DES ROCHES}

II y a 4 à $5.10^{9}$ années, une Supernova en explosant a, paraît-il, donné naissance au système solaire. Des éléments radioactifs sont apparus en cette circonstance. Ceux dont la période radioactive était très longue ont subsisté jusqu'à nos jours, ce sont essentiellement l'uranium 238, le thorium 232 et leurs descendants radioactifs, et le potassium 40. A l'origine, ces radionucléides étaient répartis de façon homogène, mais des différenciations ont eu lieu si 
bien que la croûte terrestre (épaisseur $20 \mathrm{~km}$ environ pour les continents et $50 \mathrm{~km}$ sous les océans) s'est enrichie en uranium et en thorium. Cet enrichissement fait que les concentrations de la croûte terrestre dépassent de 1 à 2 ordres de grandeur celles rencontrées dans les matériaux lunaires ou les météorites. En moyenne, la croûte terrestre contient $12 \mathrm{ppm}$ de thorium (soit $12 \mathrm{~g} / \mathrm{t}$ ) et $3 \mathrm{ppm}$ d'uranium (soit $3 \mathrm{~g} / \mathrm{t}$ ).

La plupart des roches, y compris celles d'origine sédimentaire, contiennent des quantités de cet ordre de grandeur, mais des variations par rapport à ces valeurs moyennes sont observées. C'est ainsi que les roches provenant de la fusion du manteau, comme les basaltes noirs, sont nettement plus pauvres. Ces valeurs de $12 \mathrm{~g} / \mathrm{t}$ et de $3 \mathrm{~g} / \mathrm{t}$ sont importantes et on peut les comparer par exemple aux $6 \mathrm{~g} / \mathrm{t}$ d'or que contiennent en moyenne les minerais d'or d'Afrique du Sud. Donc, si l'uranium avait une valeur comparable à celle de l'or, on pourrait exploiter directement la croûte terrestre alors que, dans les conditions économiques actuelles, on ne commence à s'intéresser qu'à des minerais d'uranium dont la teneur est de l'ordre de $300 \mathrm{~g} / \mathrm{t}$ pour ceux traités par lixiviation, et de l'ordre de 1 à $2 \mathrm{~kg} / \mathrm{t}$ pour ceux subissant une attaque chimique dans les usines de traitement. $12 \mathrm{~g} / \mathrm{t}$ de thorium 232 correspondent environ à $1 \mu \mathrm{Ci} / \mathrm{t}$ ou à $40 \mathrm{~Bq} / \mathrm{kg}$ mais avec la dizaine d'éléments de la chaîne, on aboutit à une activité totale de $400 \mathrm{~Bq} / \mathrm{kg}$; $3 \mathrm{~g} / \mathrm{t}$ d'uranium 238 correspondent aux mêmes activités si bien que la croûte terrestre a une activité moyenne globale d'environ $800 \mathrm{~Bq} / \mathrm{kg}$. Quant à l'eau des océans, elle contient très peu de thorium $\left(10^{-7} \mathrm{ppm}\right)$ et un peu plus d'uranium $\left(10^{-3} \mathrm{ppm}\right)$.

Mais si l'on s'intéresse aux zones où des phénomènes de concentration ont eu lieu, ces moyennes peuvent être localement largement dépassées; c'est ainsi que l'on trouve des zones granitiques très étendues contenant $20 \mathrm{~g} / \mathrm{t}$ d'uranium, puis des gisements uranifères de volume plus restreint dont la teneur en uranium peut prendre toutes les valeurs comprises entre quelques centaines de grammes par tonne et $700 \mathrm{~kg} / \mathrm{t}$.

\section{RADIOACTIVITÉ ATMOSPHÉRIQUE}

Dans l'atmosphère, on trouve des atomes de radon descendants de l'uranium $\left({ }^{222} \mathrm{Rn}\right)$ et du thorium $\left({ }^{220} \mathrm{Rn}\right)$ qui, au moment de leur formation au sein de la roche, peuvent par effet de recul passer dans les fluides, air ou eau, qui se trouvent dans les pores ou fissures. Véhiculés par ces fluides et soumis aux phénomènes de diffusion, ils arrivent finalement dans l'atmosphère où ils se désintègrent pour donner des atomes de descendants dont le comportement correspond à celui d'atomes d'un corps à l'état condensé qui se fixent aisément sur les surfaces solides ou liquides. Ces atomes passent ensuite à l'état de noyaux ultrafins qui s'agglomèrent et se fixent sur les particules constituant l'aérosol atmosphérique.

C'est ainsi qu'en moyenne dans l'atmosphère on trouve en régime stationnaire, au niveau du sol, environ $10^{-13} \mathrm{Ci} / \mathrm{l}\left(4 \mathrm{~Bq} / \mathrm{m}^{3}\right)$ de ${ }^{222} \mathrm{Rn}$ de période $3,8 \mathrm{j}$ et quelque $10^{-15} \mathrm{Ci} / \mathrm{l}\left(4.10^{-2} \mathrm{~Bq} / \mathrm{m}^{3}\right)$ de ${ }^{212} \mathrm{~Pb}$ de période $10,6 \mathrm{~h}$ (appelé encore thorium $B$, descendant du radon 220 de période $54 \mathrm{~s}$ ) (fig. 1 et 2). 

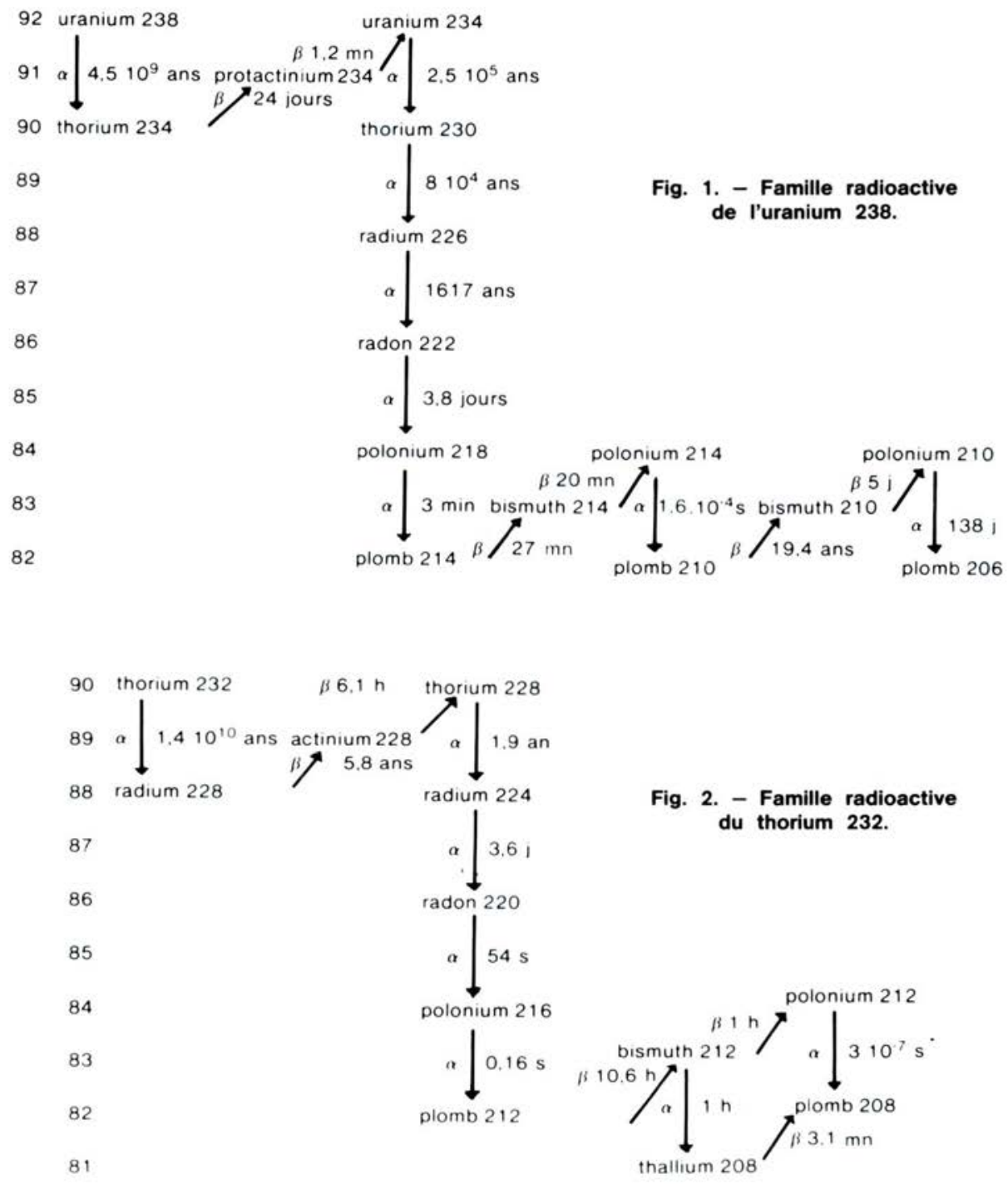

En altitude, ces concentrations décroissent en s'éloignant de la source que constitue le sol et ce d'autant plus que leur période est plus courte.

On trouve donc les radons 222 et 220 ainsi que leurs descendants en tout lieu, même dans la salle où nous sommes en ce moment. Tout le monde le sait, mais j'ai constaté qu'on avait souvent tendance à l'oublier ou tout au moins à en sous-estimer l'importance. C'est pourquoi, comme il vaut mieux voir une chose une fois que la lire cent fois, je considère que l'on 
devrait toujours faire la démonstration de la présence du radon dans l'atmosphère devant un public non initié. Comme ce n'est pas le cas aujourd'hui, je me contenterai, pour éviter les aléas de l'expérimentation, de vous décrire l'expérience proposée. Au moyen d'un aspirateur, on filtre l'air de la salle de conférence à un débit de $20 \mathrm{l} / \mathrm{min}$. Aux concentrations habituelles, par exemple $10^{-13} \mathrm{Ci} / \mathrm{l}\left(4 \mathrm{~Bq} / \mathrm{m}^{3}\right)$ de radon 222 et $4.10^{-15} \mathrm{Ci} / /(0,15$ $\mathrm{Bq} / \mathrm{m}^{3}$ ) de plomb 212 , les activités recueillies sur le filtre sont celles présentées sur la figure 3 , soit une activité d'environ $500 \alpha / \mathrm{min}$ à l'équilibre que l'on mesure au moyen d'un détecteur portatif. On peut signaler que c'est à peu près la même activité que nous avons en permanence dans nos poumons qui fonctionnent sensiblement comme le filtre.

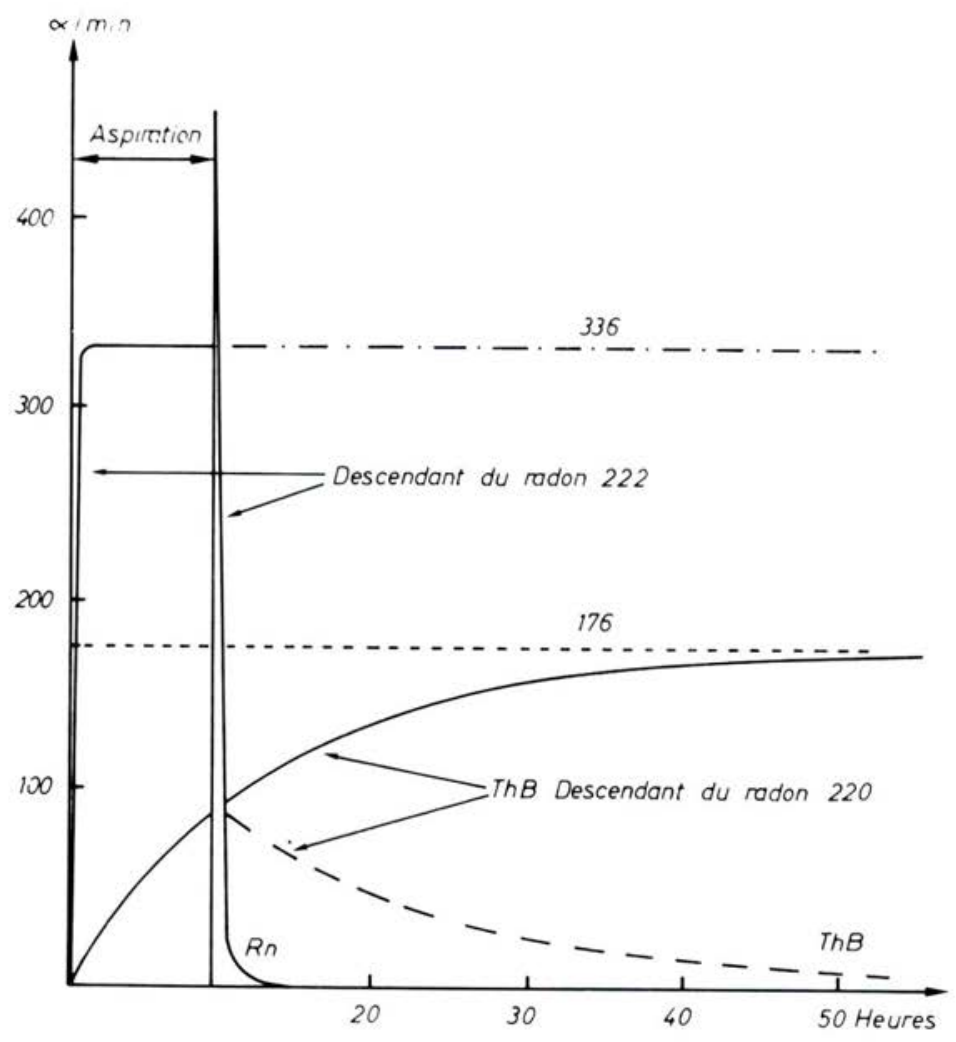

Fig. 3. - Activité recueillie sur un filtre. Prèlèvement dans l'atmosphère extérieure: $20 \mathrm{l} / \mathrm{min}$. Concentration en radon $222: 10^{-13} \mathrm{Ci} / \mathrm{l}$ à l'équilibre. Concentration en descendants du radon 220: (ThB) $4 \cdot 10^{-15} \mathrm{Ci} / \mathrm{l}$. 
Ces valeurs moyennes peuvent être localement fortement dépassées lorsque la dilution dans l'atmosphère est freinée.

C'est tout d'abord le cas des situations où l'on est en présence du phénomène bien connu d'inversion de température qui entraîne la stagnation des polluants dans les vallées. Les figures 4 et 5 représentent les résultats des mesures de la concentration en radon 222 effectuées au moyen d'un camion laboratoire circulant dans les vallées de la région du Forez. On a ainsi mis en évidence des concentrations naturelles, loin des zones d'exploitation des minerais d'uranium (fig. 5) et, de toute évidence, non liées à ces exploitations, qui atteignent, momentanément il est vrai, des valeurs dépassant $10 \mathrm{pCi} / \mathrm{l}\left(400 \mathrm{~Bq} / \mathrm{m}^{3}\right)$ à comparer aux $40 \mathrm{pCi} / \mathrm{l}\left(1500 \mathrm{~Bq} / \mathrm{m}^{3}\right)$ qui correspondent à la limite dérivée de concentration dans l'air pour les mineurs (LDCA).

C'est aussi le cas des concentrations rencontrées dans les cavités souterraines ou autres milieux fermés [8]. II faut savoir que l'air contenu dans un sol ordinaire, par exemple dans votre jardin, contient de l'ordre de $10^{4} \mathrm{~Bq} / \mathrm{m}^{3}$ de radon 222 qui constitue d'ailleurs un gaz traceur utilisé dans certaines méthodes de prospection. Un exemple des valeurs rencontrées au cours d'une campagne de prospection est donné par la figure 6. C'est pourquoi, dans les mines ou cavités diverses situées dans des formations non uranifères, on peut trouver des teneurs relativement importantes. La littérature présente de nombreuses données de concentration concernant les cavités en l'absence de tout système d'aérage autre que l'aérage naturel et pour des roches encaissantes ne présentant pas d'anomalies de teneur en uranium; les valeurs sont comprises entre 40 et $200 \mathrm{pCi} / \mathrm{l}\left(1500\right.$ et $\left.7500 \mathrm{~Bq} / \mathrm{m}^{3}\right)$. Aussi la Commission des Communautés européennes nous a demandé de mesurer les concentrations en radon et les expositions des travailleurs aux descendants du radon dans des mines européennes non-uranifères [1].

Une campagne de mesures réalisée dans huit mines a montré que dars quatre mines (charbon et potasse) les équivalents de dose ne dépassaient pas la limite annuelle de $5 \mathrm{mSv}$ pour les travailleurs non directement affectés à des travaux sous rayonnements. Par contre, dans quatre autres mines, cette limite était dépassée. Dans ces dernières, la valeur moyenne était de 4,5 - 8,7 - 15 et $17,5 \mathrm{mSv}$. L'exposition maximale correspondrait à $25 \mathrm{mSv}$. La figure 7 représente la distribution des expositions dans ces huit mines. L'exposition externe et l'exposition interne due aux poussières à vie longue qu'il y aurait lieu d'ajouter modifieraient peu le résultat.

Ces résultats ont conduit la Commission des Communautés européennes à demander la poursuite de l'étude sur un nombre plus important de mines et de chantiers souterrains [5]. On a ainsi déterminé que la dose pouvait être comprise entre 0,5 et $1,2 \mathrm{mSv}$ dans le métro parisien, 


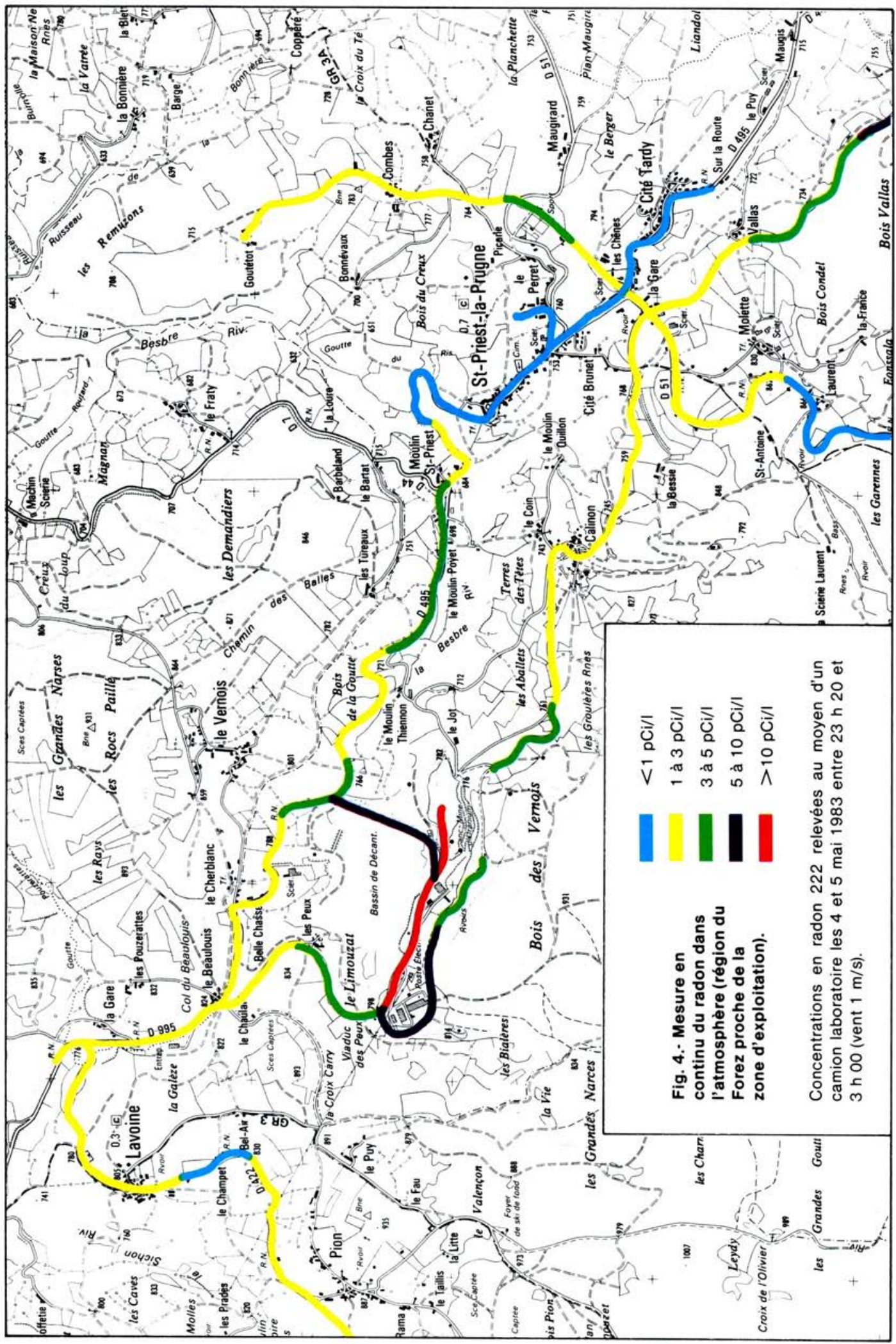




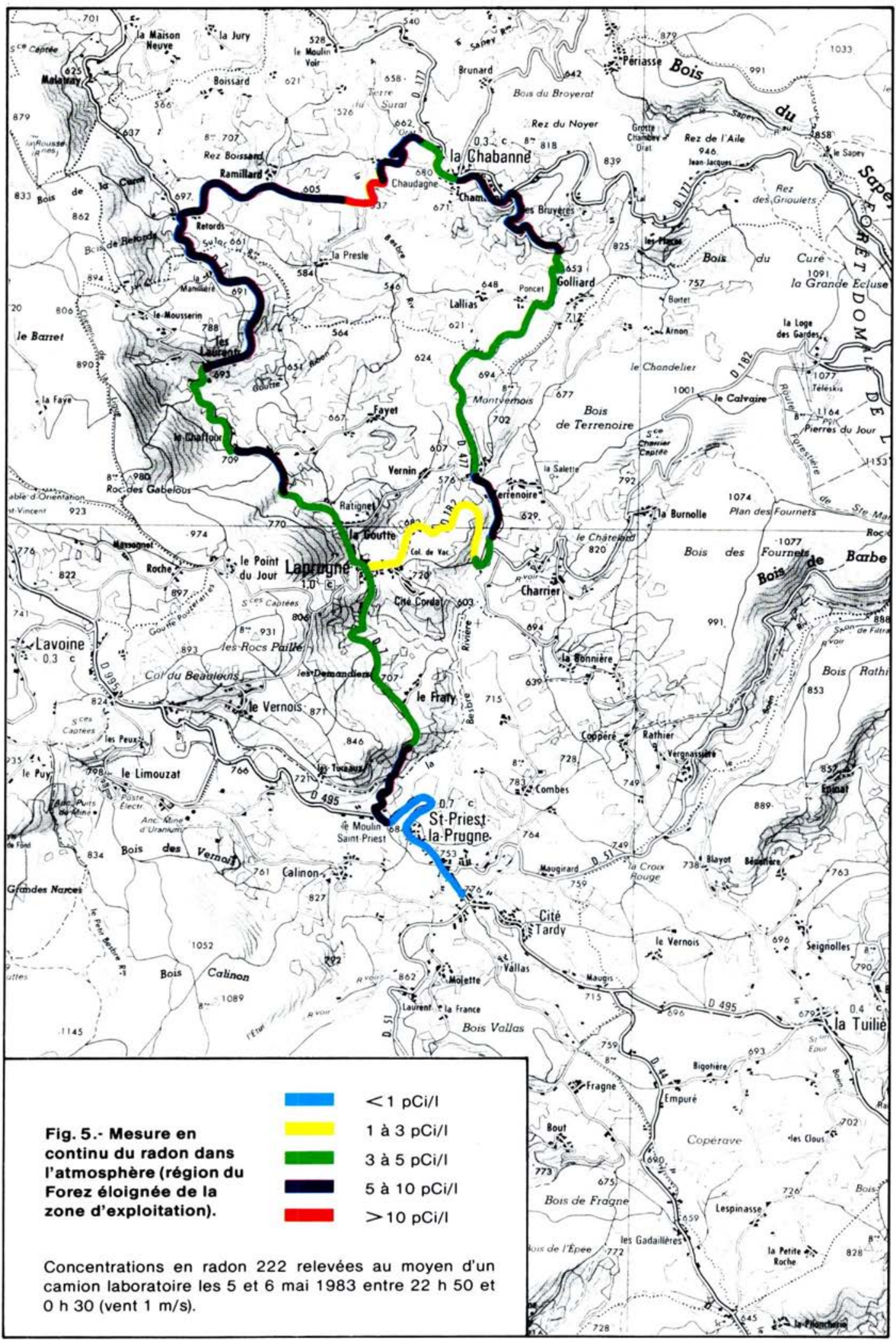




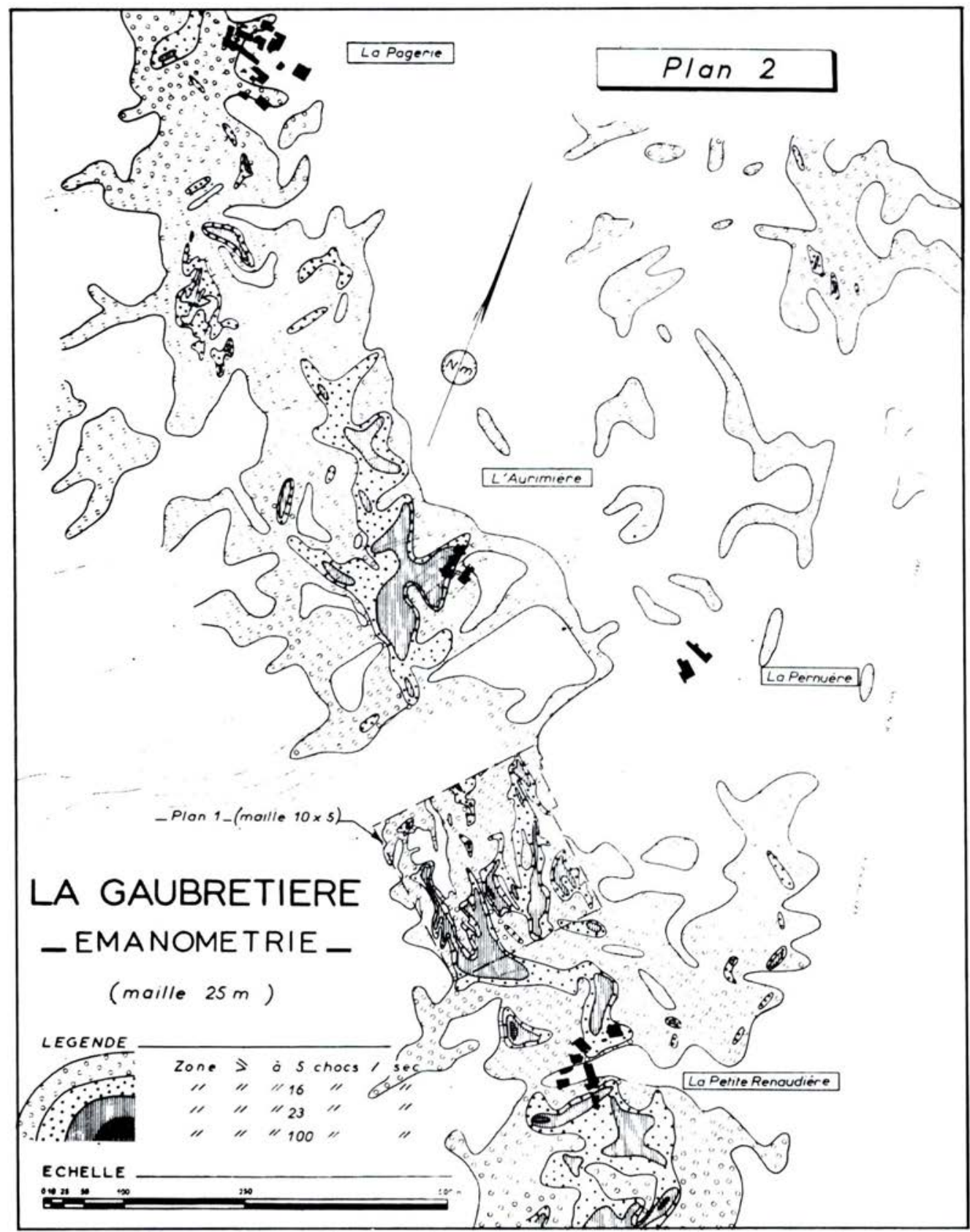

Fig. 6. - Concentration en radon dans l'air du sol $(0,1$ choc par seconde correspond approximativement à $1500 \mathrm{~Bq} / \mathrm{m}^{3}$ soit la concentration moyenne maximale dans les mines d'uranium). 


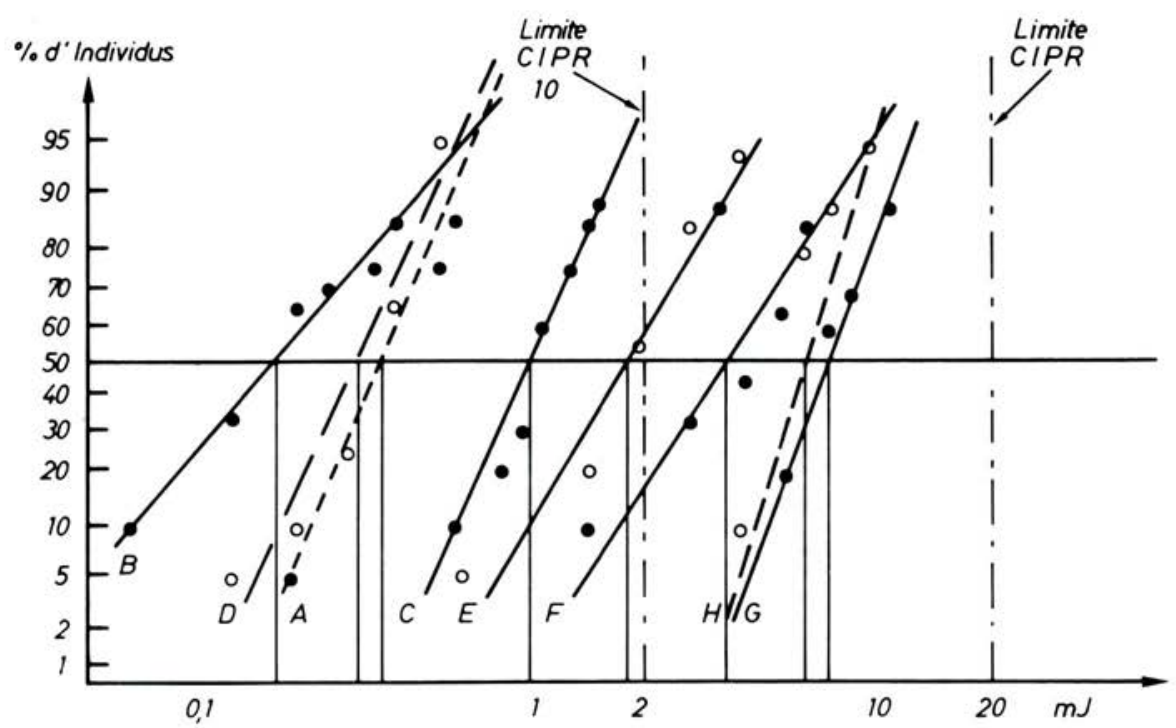

Fig. 7. - Distribution des quantités annuelles d'énergie $\alpha$ inhalée (personnel de 8 mines non uranifères).

entre 0,05 et 0,32 mSv dans le laboratoire de Modane et le tunnel de Fréjus, entre 1,2 et $2,2 \mathrm{mSv}$ dans des champignonnières, ces expositions étant évaluées pour une présence de $2000 \mathrm{~h}$. Dans les quarante mines étudiées, la moitié d'entre elles pouvaient conduire à des expositions annuelles supérieures à $5 \mathrm{mSv}$. Des valeurs nettement plus importantes pouvant atteindre la centaine de millisieverts ont été trouvées par ailleurs dans un tunnel non ventilé.

En ce qui concerne les niveaux de concentration à l'intérieur des habitations, on peut se reporter aux campagnes de mesures systématiques entreprises par le Département de protection sanitaire du CEA depuis 1982 [3, 9, $14,15]$. Dans le Limousin, 96 prélèvements ont été réalisés en 19 lieux donnant, pour chaque lieu, des valeurs moyennes comprises entre 7,4 et 277,5 $\mathrm{Bg} / \mathrm{m}^{3}$ de radon et des valeurs maximales comprises entre 18,5 et $851 \mathrm{~Bq} /$ $\mathrm{m}^{3}$. On calcule que les concentrations précédentes conduiraient à des doses moyennes comprises entre 0,17 et 6,5 mSv et des doses maximales comprises entre 0,44 et $20 \mathrm{mSv}$. Avec les mêmes correspondances, les résultats des 751 mesures réalisées dans 20 départements et dans la région parisienne ont donné des valeurs moyennes comprises entre 0,45 et 2,7 $\mathrm{mSv}$ et des valeurs maximales comprises entre 2,1 et $30 \mathrm{mSv}$.

\section{CAS DES MINES D'URANIUM [2, 11 - 13]}

Dans le cas des mines, on change évidemment d'échelle puisque l'on passe de quelques grammes d'uranium par tonne de roches à quelques kilogrammes voire centaines de kilogrammes par tonne.

VOL. $22 \cdot \mathrm{N}^{\circ}{ }_{4}$ 
II faut retenir les ordres de grandeur suivants:

- irradiation externe: dans une galerie creusée dans un minerai à $0,5 \%$, ou au-dessus d'une surface plane constituée de minerai à $1 \%$ (cas des mines à ciel ouvert), on a un niveau d'irradiation correspondant à $2,5.10^{-2} \mathrm{mSv} / \mathrm{h}$ eure, soit $50 \mathrm{mSv} / \mathrm{an}$, valeur limite pour un travailleur exposé $2000 \mathrm{~h}$; pour des teneurs différentes, les expositions sont pratiquement proportionnelles à cette teneur;

- la concentration en radon 222 est beaucoup plus difficile à prévoir, elle dépend tout à la fois de la teneur en uranium, de la fracturation des terrains, de l'importance des eaux circulant dans les fractures et véhiculant le radon jusqu'aux cavités et enfin de l'importance et de la qualité de la ventilation; pratiquement, elle peut varier entre 0,1 et $10^{3}$ fois la LDCA pour les mineurs, soit $1500 \mathrm{~Bq} / \mathrm{m}^{3}$ pour le radon en équilibre avec ses descendants;

- la concentration en poussières à vie longue (particules de minerai mises en suspension dans l'air) peut, elle aussi, varier, mais de façon moins importante entre 0,1 et 10 LDCA.

On se trouve donc devant un problème réel d'exposition des travailleurs, problème qui est et doit être traité avec une grande rigueur. Fort heureusement, dès le début de l'exploitation minière en France, approximativement dix ans avant la plupart des autres pays, un effort important pour améliorer les conditions de travail a été entrepris, ce qui a permis d'éviter les conséquences sanitaires malheureuses constatées à l'étranger [13].

Une réglementation stricte a été établie dès 1958. En 1987, elle a été révisée pour la deuxième fois, mais sans qu'en soient changées les caractéristiques essentielles qui étaient et restent le suivi individuel et le respect d'une norme cumulant les différents types d'exposition.

La réglementation actuelle repose sur le respect de la formule suivante:

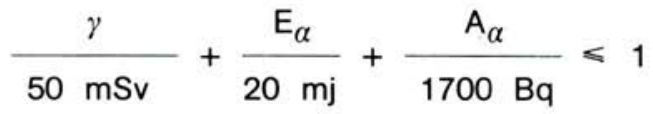

$\begin{array}{ll}\gamma & =\text { dose due à l'irradiation externe } \\ \mathrm{E}_{\alpha} & =\text { énergie potentielle } \alpha \text { des descendants du radon } 222 \text { (en millijoules) } \\ \mathrm{A}_{\alpha} & =\text { activité } \alpha \text { à vie longue des poussières. }\end{array}$

La mise au point en 1982 d'un dosimètre individuel fournissant les trois données $\gamma, \mathrm{E}_{\alpha}$, et $\mathrm{A}_{\alpha}$, a permis de suivre avec une bonne précision l'exposition de chaque mineur et d'atteindre les objectifs fixés par la nouvelle réglementation pour l'ensemble du personnel fond dès 1985 (fig. 8) [10].

Je voudrais également vous donner quelques informations relatives aux gisements mondiaux de très forte teneur comme le gisement de Cigar Lake au Canada. II s'agit d'une lentille contenant 110000 t d'uranium située 


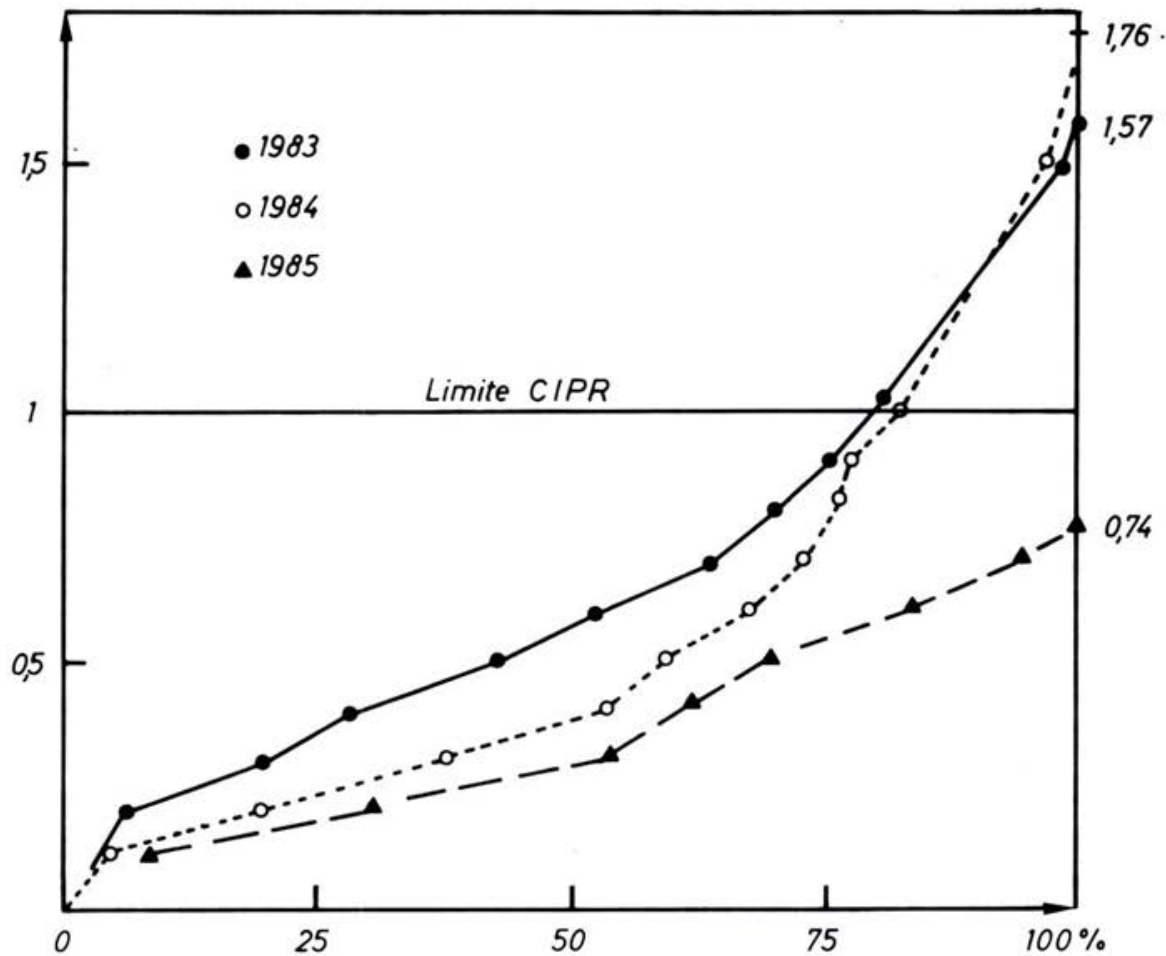

Fig. 8. - Répartition de l'effectif "fond" en fonction de la valeur du cumul des équivalents de dose reçus.

à une profondeur de $400 \mathrm{~m}$, de largeur 20 à $100 \mathrm{~m}$, de longueur $800 \mathrm{~m}$ (soit en gros la même surface que les Champs Elysées) et de hauteur 5 à $30 \mathrm{~m}$ [7]. Etant donné la profondeur du gisement, l'exploitation en mine à ciel ouvert n'est pas concevable, d'où la décision d'envisager des méthodes particulières d'exploitation pour résoudre le problème de l'exposition des mineurs en présence de minerai dont la teneur est de $12 \%$ et peut même atteindre localement 50 , voire $70 \%$. II faut savoir que, dans ce cas, au centre des galeries tracées dans le minerai, les expositions externes atteignent les valeurs suivantes:

- minerai à $12 \% \rightarrow 0,6 \mathrm{mSv} / \mathrm{h}$

- minerai à $70 \% \rightarrow 3,5 \mathrm{mSv} / \mathrm{h}$

La figure 9 présente les trois méthodes envisagées adaptées à une exploitation de ce gisement en terrain argileux:

- utilisation de forage de grand diamètre par réalésage ascendant d'un forage guide;

- utilisation de l'hydrofraise de Solétanche qui réalise un trou borgne, la paroi de la saignée étant maintenue par des boues;

- utilisation d'une méthode conventionnelle avec des matériels télécommandés. 


\section{Blind Drilling Method (doc. CLMC)}

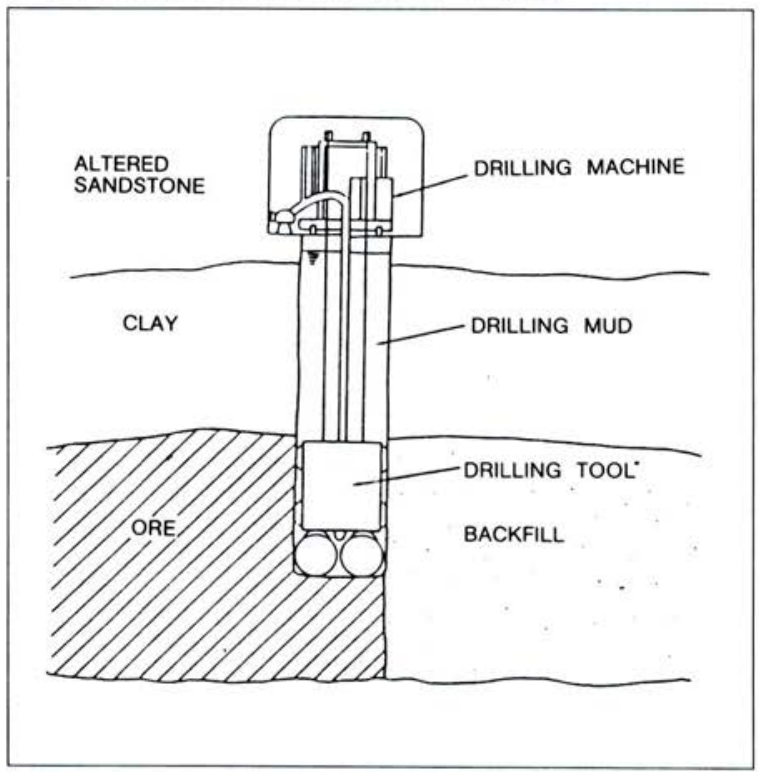

Raise Boring Method (doc. CLMC)

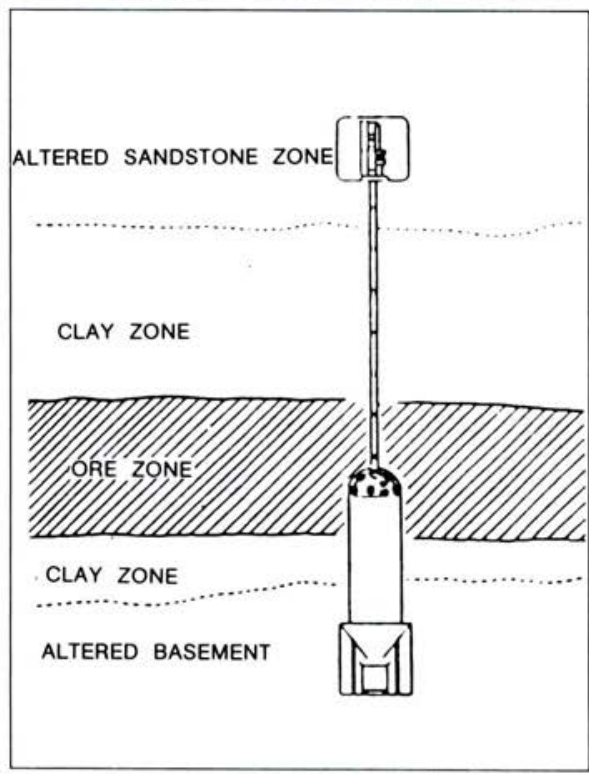

\section{Undercut and Fill Method (doc. CLMC)}
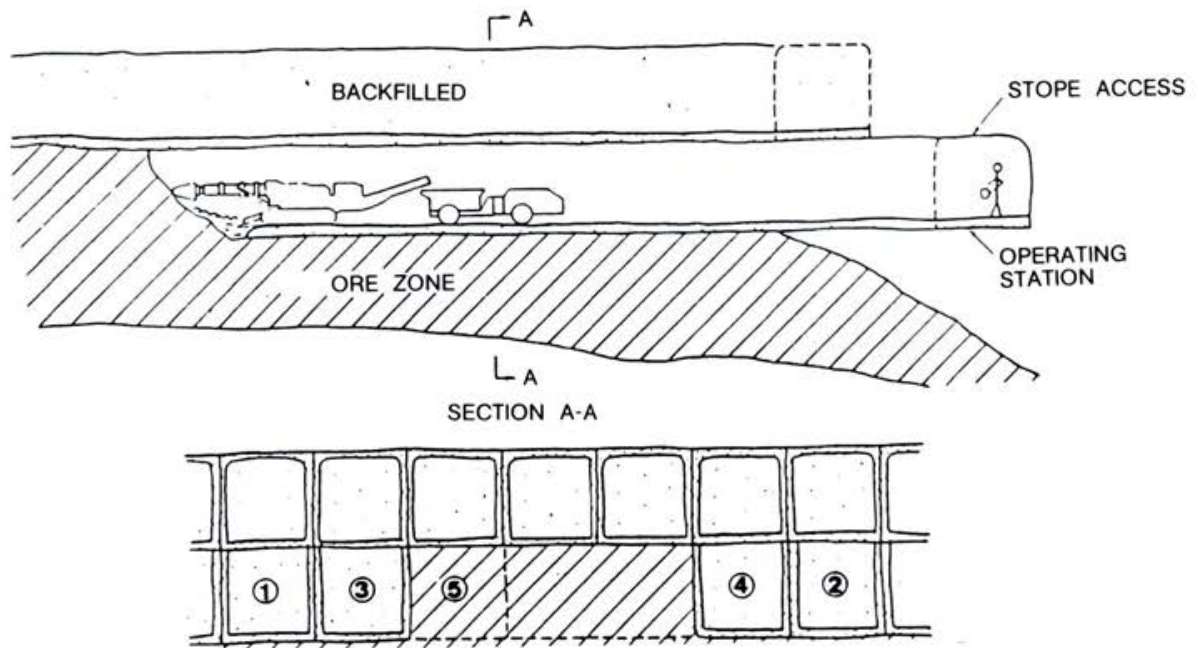

Fig. 9. - Méthodes possibles pour exploiter les minerais d'uranium à très forte teneur, extrait de [7]. 


\section{APPLICATION A LA GESTION DES DÉCHETS}

On peut tirer parti de ces données en les utilisant comme guide pour choisir des limites d'exposition tolérables pour les générations futures dans les scénarios retenus pour évaluer les nuisances présentées par les stockages de déchets. En effet, on peut se demander s'il serait bien raisonnable et économiquement judicieux de vouloir à tout prix se fixer des limites d'exposition très inférieures aux valeurs correspondant à des scénarios semblables et au moins aussi probables, mais impliquant la radioactivité naturelle.

Si nous examinons le cas des centres de stockage en surface, on peut comparer le scénario d'implantation d'une habitation sur ce centre et les implantations sur une zone minéralisée comme il en existe beaucoup en France. On constate que la limite de $1 \mathrm{mSv}$ par an adoptée pour les centres de stockage, qui correspond sur la figure 10 à environ 10 chocs/seconde (c/s) pour une exposition de 7000 heures par an, est dépassée systématiquement dans toutes les zones en couleur et que l'on peut atteindre fréquemment des valeurs trente fois plus élevées. A noter encore qu'il conviendrait d'ajouter l'exposition au radon qui, sans aucun doute, peut atteindre $10 \mathrm{mSv}$, voire quelques centaines de millisieverts dans ces zones.

Si nous examinons le cas d'un stockage géologique en profondeur, on peut comparer les différents scénarios d'intrusion à celui correspondant au séjour dans une mine riche au voisinage des zones minéralisées. Dans ce cas, le risque radon peut devenir colossal si l'on suppose que les intrus sont ignorants des risques provenant de la radioactivité. II est tout à fait possible et probable dans une zone fortement minéralisée, dont la fréquence d'existence est au moins aussi importante que celle des stockages de haute activité, d'être exposé à des concentrations en radon de 1000 à 10000 fois la LDCA pour les mineurs. II en résulte des expositions de 25 à $250 \mathrm{mSv} / \mathrm{h}$ avec des limites théoriques de plusieurs dizaines de sieverts par heure. On peut comparer la valeur de $250 \mathrm{mSv}$, associée à l'une de ces cavités prise comme référence, au risque présenté par un stockage de verre contenant des produits de fission.

L'irradiation $\gamma$ provenant d'un séjour devant un mur de déchets vitrifiés de haute activité supposé de dimensions infinies devient, au bout de quelque $10^{3}$ années de stockage, inférieure à celle découlant du même séjour dans la cavité. En pratique, une colonne de conteneurs de ce type de déchets ne doit pas entraîner, même au début du stockage, une exposition supérieure (fig. 11).

L'inhalation pendant une journée entière de poussières de verre à raison de $10 \mathrm{~m}^{3}$ d'air à la concentration de $0,1 \mathrm{mg} / \mathrm{m}^{3}$ (cas extrême très exagéré car la poussière ne contiendra qu'un pourcentage limité de verre) correspondra également à une exposition inférieure à celle résultant de séjours dans la cavité d'une durée de 10 heures ou de 1 heure au bout de $10^{3}$ et $10^{4}$ années respectivement (fig. 12). On peut aussi retenir que, dans des cas moins extrêmes mais très probables, un séjour dans une cavité mal ventilée peut entraîner des irradiations de $2,5 \mathrm{mSv} / \mathrm{h}$ (concentration en radon 222, de $100.10^{-10} \mathrm{Ci} / \mathrm{l}$ $\left(4.10^{5} \mathrm{~Bq} / / \mathrm{m}^{3}\right)$ soit 250 LDCA pour les travailleurs).

Sans vouloir en tirer des conclusions définitives, on ne peut ignorer ces valeurs guides. 


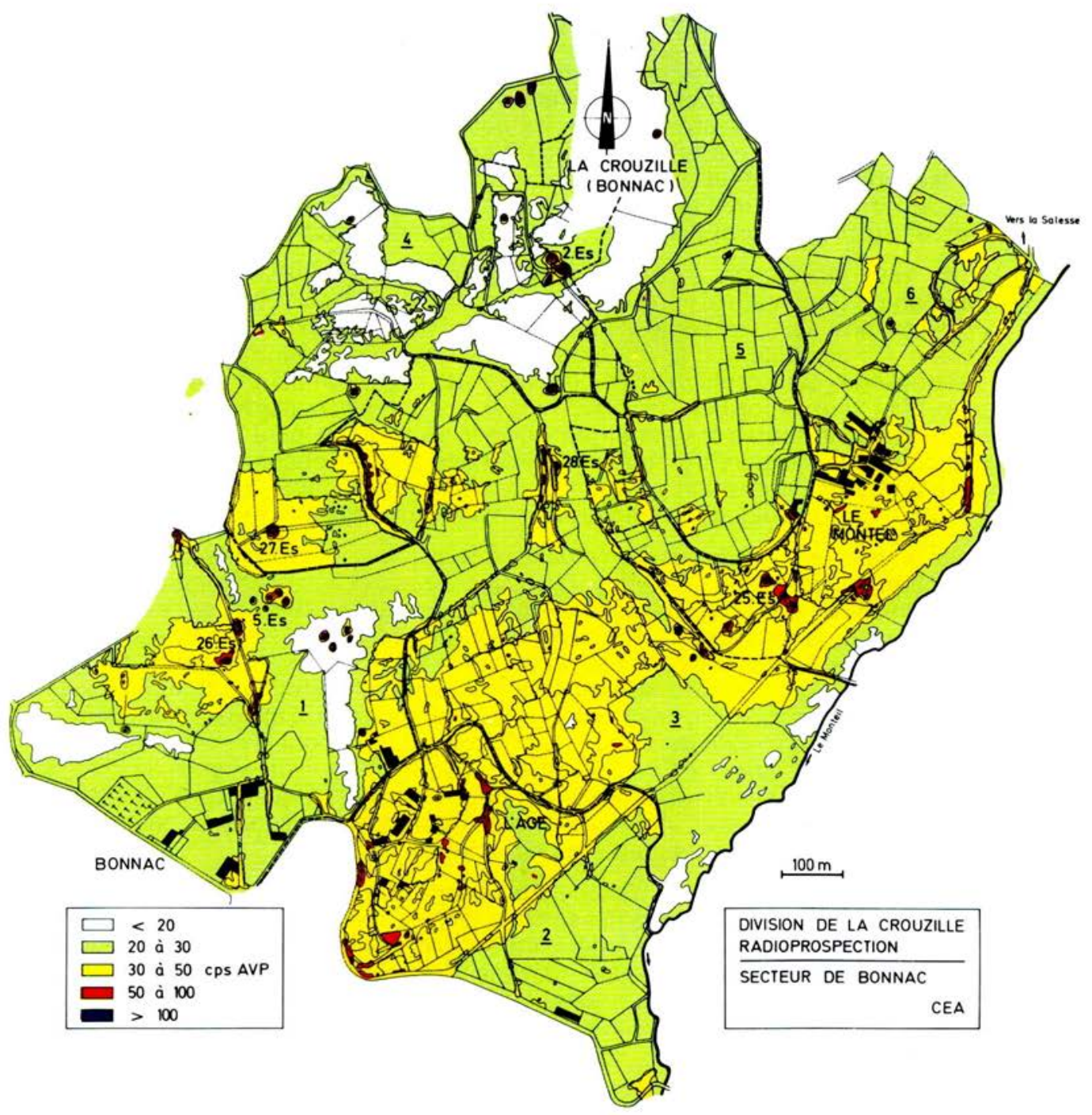

Fig. 10. 


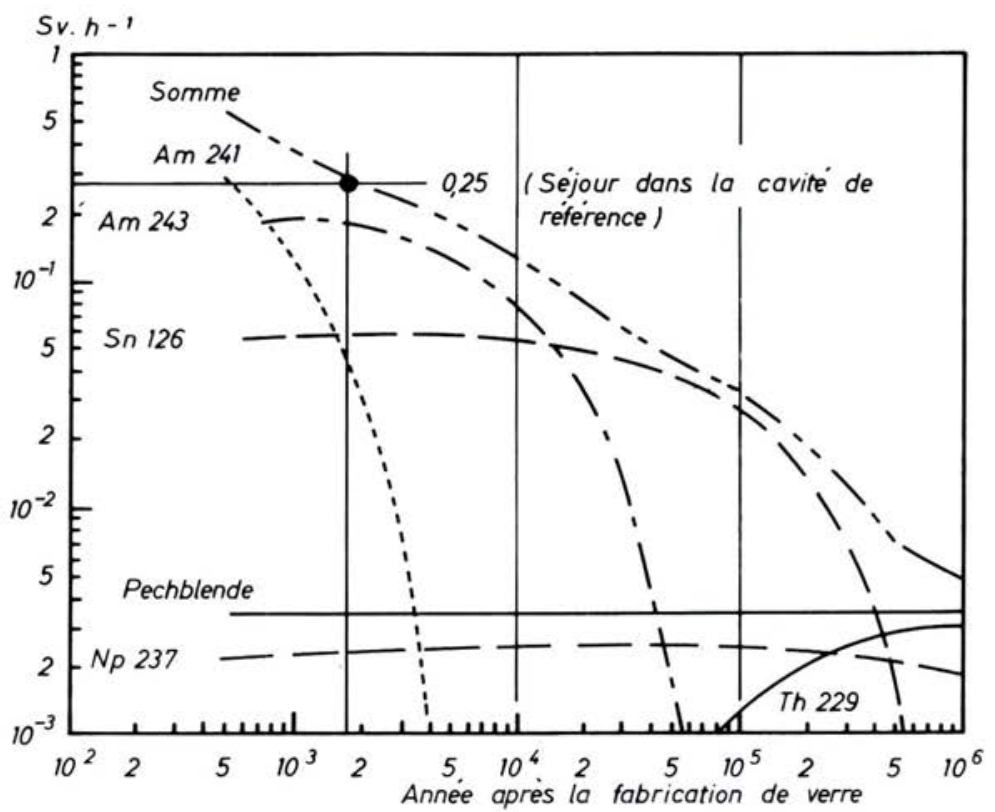

Fig. 11. - Débit de dose près d'une masse semi-infinie de déchets vitrifiés de haute activité.

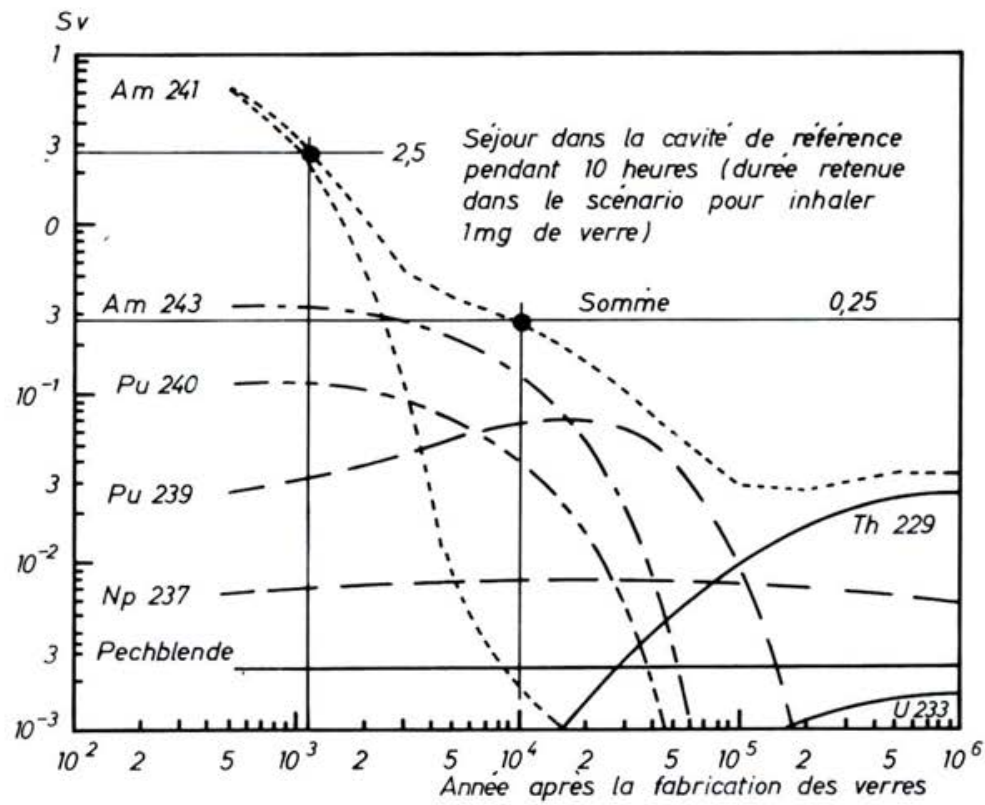

Fig. 12. - Dose due à l'inhalation de $1 \mathrm{mg}$ de déchets vitrifiés de haute activité. 


\section{CONCLUSIONS}

Dans le système solaire et, en particulier, sur notre planète, tous les matériaux sont naturellement radioactifs, que ce soit la terre, les eaux des rivières et des océans ou de sources dites pures, le charbon, le pétrole, les engrais..., l'air de nos montagnes ou celui de nos salles de réunion. Dans une cavité creusée dans le sol et mal ventilée comme le sont certaines mines non uranifères ou comme l'ont été les habitations de certains amateurs de la nature dits "écologistes", on trouve facilement autant de radon que dans nos mines d'uranium. On peut en trouver beaucoup plus dans des conditions particulières correspondant à des cavités mal ventilées situées dans des zones uranifères. Si toutes les données de la Commission internationale de protection radiologique sont exactes, des effets non stochastiques peuvent exister pour des individus séjournant quelques heures en de tels lieux, ce qui à notre connaissance n'a jamais été mis en évidence. Cette grande variabilité dans les concentrations pouvant exister en milieu souterrain laisse aussi planer quelques doutes sur la validité de la dosimétrie utilisée dans les enquêtes épidémiologiques pour évaluer l'exposition des mineurs dans les exploitations très anciennes pour lesquelles on ne possède que très peu de résultats de mesure.

Cependant, même s'il est vrai que les mineurs d'uranium sont exposés à des niveaux relativement élevés par rapport à beaucoup de travailleurs de l'industrie nucléaire, il faut noter que leur exposition, lorsque les dispositions adéquates de protection sont prises, se rapproche de celle correspondant à de nombreux travailleurs ou même de membres de la population du fait de la radioactivité naturelle.

On doit aussi retenir que la radioactivité naturelle délivre des doses collectives bien supérieures à celles provenant de la radioactivité artificielle. Je rappellerai simplement l'évaluation faite par $M$. PRETRE qui arrive à la conclusion qu'en Suisse la radioactivité naturelle équivaut à dix accidents de Tchernobyl par an. Ceci peut être considéré comme rassurant quant à l'avenir de l'humanité évoluant dans un monde nucléaire, surtout si l'on prend en considération l'exposition à laquelle ont été soumis nos ancêtres vivant dans les cavernes.

Malgré l'effort de ces quelques dernières années, la radioactivité naturelle est encore insuffisamment connue. Les enseignements en radioprotection devraient lui consacrer une part très importante et comprendre des travaux pratiques incluant la mesure de l'activité atmosphérique: croissance et décroissance de l'activité recueillie sur un filtre avec identification des descendants du radon 222 et 220 . Cette formation me paraît d'autant plus nécessaire qu'avec l'apparition des détecteurs performants, utilisant des techniques d'élimination du "bruit de fond radon", les futures générations risquent d'oublier l'existence de ces radionucléides naturels.

En conclusion, je dirai que, de toute façon, il ne faut jamais oublier que la nature réserve aux générations futures des risques nombreux, notamment ceux dus à la présence de la radioactivité naturelle, plus importants que ceux provenant de l'utilisation de l'énergie nucléaire, même en tenant compte des stockages de déchets. Cependant, l'ensemble de ces risques 
demeure tout à fait maîtrisable par une société scientifiquement et techniquement évoluée.

Je ne peux enfin résister à la tentation de vous rappeller deux citations. La première dont j'ignore l'auteur:

"Larbuste de la radioactivité artificielle a beau pousser au milieu de la forêt de

la radioactivité naturelle, les yeux du monde restent encore fixés sur lui", est assez représentative de la situation actuelle, et la deuxième, de Paul Valèry:

"Ce qui est simple est toujours faux, ce qui ne l'est pas est inutilisable", illustre bien pourquoi il est si difficile de faire passer dans le public nos propres convictions quant au bien-fondé du choix de l'utilisation de l'énergie nucléaire".

\section{TABLEAU RÉCAPITULATIF DES PRINCIPALES DONNÉES}

\section{RADIOACTIVITÉ NATURELLE DES ROCHES}

Teneur moyenne des roches : uranium $3 \mathrm{~g} / \mathrm{t}$, thorium $12 \mathrm{~g} / \mathrm{t}$

Teneur en uranium des minerais : $300 \mathrm{~g}$ à $700 \mathrm{~kg} / \mathrm{t}$

Teneur moyenne en or des minerais d'or: $6 \mathrm{~g} / \mathrm{t}$

Activité moyenne des roches : $800 \mathrm{~Bq} / \mathrm{kg}$

[Niveau de tolérance pour les denrées alimentaires suite à

Tchernobyl (Conseil des Communautés européennes): $600 \mathrm{~Bq} / \mathrm{kg}$ ].

II. CONCENTRATIONS EN RADON OU EXPOSITIONS ANNUELLES

\section{CORRESPONDANTES}

Limite dérivée de concentration en radon pour les mineurs (LDCA):

$1500 \mathrm{~Bq} / \mathrm{m}^{3} \rightarrow 50 \mathrm{mSv} / \mathrm{an}$ pour $2000 \mathrm{~h}$ de travail

Atmosphère

Air du sol

Cavités mal ventilées

Mines et chantiers souterrains

Métro

Modane et Fréjus

Champignonnières

4 à $400 \mathrm{~Bq} / \mathrm{m}^{3}$

1000 à $10^{7} \mathrm{~Bq} / \mathrm{m}^{3}$

1500 à $7500 \mathrm{~Bq} / \mathrm{m}^{3}$

: 4,50 à $25 \mathrm{mSv}$

: 0,50 à $1,20 \mathrm{mSv}$

0,05 à $0,32 \mathrm{mSv}$

Maisons en France, moyenne

1,20 à $2,20 \mathrm{mSv}$

Maisons en France, maximum

0,17 à $6,5 \mathrm{mSv}$

0,44 à $30 \mathrm{mSv}$

\section{EXPOSITION DANS LES MINES D'URANIUM}

\section{Irradiation externe}

Galerie dans un minerai à $0,5 \%$ ou mine à ciel ouvert dans un minerai à $1 \%$

Galerie dans un minerai très riche $12 \%$

$70 \%: 3,5 \mathrm{mSv} / \mathrm{h} \rightarrow 3,5 \mathrm{~Sv} / \mathrm{an}$

\section{Irradiation interne}

Mines ventilées

Mines riches en milieu confiné

$$
\begin{aligned}
& 0,025 \mathrm{mSv} / \mathrm{h} \\
& \text { pour } 2000 \mathrm{~h} \text { de travail } \\
& 20
\end{aligned}
$$$$
\begin{aligned}
& 0,6 \mathrm{mSv} / \mathrm{h} \rightarrow 1,2 \mathrm{~Sv} / \mathrm{an} \\
& 3,5 \mathrm{mSv} / \mathrm{h} \rightarrow 3,5 \mathrm{~Sv} / \mathrm{an}
\end{aligned}
$$

0,1 à $10^{3}$ LDCA $\rightarrow 0,025$ à $25 \mathrm{~Sv} / \mathrm{h}$

$10^{3}$ à $10^{4}$ voire $10^{6}$ LDCA

- 25 à $250 \mathrm{mSv} / \mathrm{h}$ voire $25 \mathrm{~Sv} / \mathrm{h}$

Cavité de référence (non ventilée en zone uranifère) $: 10^{4}$ LDCA $\rightarrow 250 \mathrm{mSv} / \mathrm{h}$ 


\section{BIBLIOGRAPHIE}

[1] BERNHARD S., LE GAC J., SEGUIN H., ZETTWOOG P. Radon levels and radon daughter exposures of workers in non-uranium mines of the E.C. In: International conference on radiation hazards in mining, Golden, Colorado, USA, Oct. 4-9, 1981 (GOMEZ M. Ed.) New York: American institute of mining, metallurgical and petroleum engineers, 1981, 625-628.

[2] BERNHARD S., ZETTWOOG P. La radioprotection dans les mines d'uranium françaises. In: Société française d'énergie nucléaire (SFEN). Les techniques d'exploitation et de traitement des minerais d'uranium, Paris, 17 avril 1986. Paris: SFEN, 1986, 96-116.

[3] BOUVILLE A. Différentes composantes de la radioactivité naturelle et les fluctuations selon le lieu. In: Société française de radioprotection (SFRP). Les données actuelles sur la radioactivité naturelle, Monte-Carlo, 5-7 nov. 1984. Radioprotection, 1985, 20 (1) 21-31.

[4] DOUSSET M., JAMMET $H$. Les sources naturelles d'exposition aux rayonnements ionisants. Clefs CEA, 1986, $n^{\circ} 3,15-31$.

[5] Evaluation des doses dues au radon 222 et aux produits de filiation recues par les travailleurs dans les chantiers et ateliers souterrains. Rapport CEA/IPSN/DPT/ SPIN/LRPM, n 015/LRPM (2), 1986.

[6] FRANÇOIS H. "La radioactivité c'est naturel"... Monaco: Editions du Rocher, 1983.

[7] LIEGEOIS E. Cigar Lake: Comment exploiter un tel gisement ? Energie Magazine, oct.-nov. 1986, 9-13.

[8] LE GAC J., ZETTWOOG P. Radioactivité de l'air dans les mines souterraines et les cavités naturelles. In: Société française de radioprotection (SFRP). Les données actuelles sur la radioactivité naturelle, Monte-Carlo, 5-7 nov. 1984. Fontenay-aux-Roses: SFRP, 1985, 130-160.

[9] MADELMONT C., RANNOU A., RENOUARD H. Sources externes: cosmique, tellurique et domestique. In: Société française de radioprotection (SFRP). Les données actuelles sur la radioactivité naturelle, Monte-Carlo, 5-7 nov. 1984. Radioprotection, 1985, 20 (1) 33-44.

[10] OCDE-AEN. Dosimetry aspects of exposure to radon and thoron daughter products. Aspects dosimétriques de l'exposition aux produits de filiation du radon et du thoron. Paris: OCDE, 1983.

[11] PRADEL J. La prospection de l'uranium par le radon. Rapport CEA $n^{\circ} 588,1956$.

[12] PRADEL J., BILLARD F., MIRIBEL J., GANGLOFF A., PUIBARAUD Y., TAYEB G. La prospection de l'uranium par le radon. Rapport CEA $n^{\circ} 2330,1963$.

[13] PRADEL J., ZETTWOOG P. Hier et maintenant: la radioprotection dans les mines d'uranium. Rev. Gen. Nucl., 1984, $\mathrm{n}^{\circ} 1,38-57$.

[14] RANNOU A. Indoor radon exposure assessment: an overview of the French survey. 2. International speciality conference on indoor radon, Cherry Hill, New Jersey, USA, April 6-10, 1987.

[15] RANNOU A., MADELMONT C., RENOUARD H., JEANMAIRE L., TYMEN G., MOUDEN A. Résultats de surveillance du radon à l'intérieur des habitations. In: Société française de radioprotection (SFRP). Les données actuelles sur la radioactivité naturelle, Monte-Carlo, 5-7 nov. 1984. Fontenay-aux-Roses: SFRP, 1985, 93-113.

[16] Société française de radioprotection (SFRP). Les données actuelles sur la radioactivité naturelle, Monte-Carlo, 5-7 nov. 1984. Fontenay-aux-Roses: SFRP, 1985. 